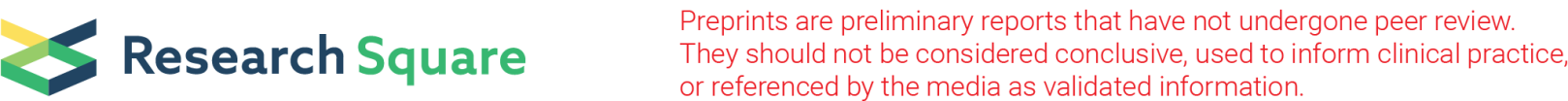

\section{The effects of Qigong intervention based on internet on quality of life and physical fitness in Chinese postoperative breast cancer patients: a protocol of randomized controlled trial}

Lina Yu

shandong normal university https://orcid.org/0000-0003-4035-9918

Xiaosheng Dong

Shandong University

Kai Yuan

Shandong Qianfoshan Hospital

Xiangren Yi

Shandong University

Yuanlong Shen

shandong normal university

Meng Ding ( $\nabla$ dingmeng@sdnu.edu.cn )

Shandong Normal University

Study protocol

Keywords: qigong intervention, postoperative breast cancer patients, Qigong-Baduanjin, postoperative exercise rehabilitation

Posted Date: September 2nd, 2020

DOI: https://doi.org/10.21203/rs.2.20983/v2

License: (c) (i) This work is licensed under a Creative Commons Attribution 4.0 International License. Read Full License 


\section{Abstract}

Background: The purpose of this study is to verify the improvement of remote qigong intervention on the quality of life and physical fitness of breast cancer patients after surgery by means of a randomized controlled trial, and to compare it with the conventional exercise combination of aerobic exercise and resistance training.

Methods/design: The research approach applied in this study is a randomized controlled trial. After completing the baseline questionnaire and physical fitness test, participants were randomly assigned to either the qigong group or the combined exercise rehabilitation group. Patients in the qigong group performed Qigong-Baduanjin twice a week for 30 minutes each time under remote guidance and practiced Baduanjin by themselves at other times. Patients in the combined exercise rehabilitation group were given resistance training twice a week for 30 minutes under remote guidance, and walking the rest of the time. At the end of the 12-week intervention, outcomes testing and data collection were carried out. The primary outcomes are quality of life, measured using the Medical Outcomes Study 36-Item ShortForm Health Survey (SF-36), and the Functional Assessment of Cancer Therapy-Breast (FATC-B). The secondary outcomes include cardiopulmonary endurance, upper limb strength, lower limb strength and skinfold thickness.

Discussion: The importance of postoperative exercise rehabilitation for breast cancer has been gradually accepted by more and more doctors and patients, but further research and development of simple and practical means of exercise rehabilitation are necessary. Remote qigong intervention for breast cancer patients via the Internet will be a great alternative.

Trial registration: chictr, ChiCTR1900027989 December 07,2019 http://www.chictr.org.cn/index.aspx

\section{Background}

Breast cancer is the most common malignant tumor in women worldwide and the second leading cause of cancer death in women. In recent years, with the continuous improvement of medical technology, the number of breast cancer survivors continues to grow. However, postoperative breast cancer patients often suffer from complications such as pain, limited upper limb activity, fatigue, obesity, premature menopause, lymphedema, which seriously reduces the quality of life of patients. Therefore, more and more attention has been paid to the rehabilitation of postoperative breast cancer patients.[1-2]

Previous studies have revealed that exercise intervention will reduce mortality risk in breast cancer patients and improve the quality of life, physical function and social cognition of survivors after breast cancer surgery [3-4], and has a certain healing effect on patients after breast cancer surgery [5-6]. Nevertheless, traditional exercise intervention failed to attract participation and improve rehabilitation effect because of distance, time consumption, money, transportation and so on. With the development of modern network technology, the remote intervention has become possible. Studies have verified the effectiveness of remote intervention for breast cancer patients after surgery. 
Qigong is a form of exercise focusing on physical activity, breathing and psychological regulation, and a psychosomatic therapy [15]. Previous studies have shown that Qigong exercise can improve the fatigue perception and sleep quality of breast cancer patients undergoing postoperative chemotherapy, reduce the risk of lymphedema, and improve their quality of life and physical fitness[7]. However, there is still a lack of research on the effect of remote exercise Qigong intervention on postoperative recovery of breast cancer patients and it is also unclear whether Qigong intervention is more advantageous than conventional intervention combination of aerobic exercise and resistance exercise. This study is intended to verify the effect of long-range Qigong intervention on the improvement of quality of life and physical fitness in breast cancer patients by means of randomized controlled trials and to compare it with conventional exercise intervention methods.

\section{Design And Methods}

\section{Design}

This study is a RCT with an intervention time of 12 weeks. Trial participants will be recruited starting in February 2019 and the data will be analyzed after the sample size is achieved according to the study design. The flow chart of the study design is shown in Fig 1.

\section{Subject Recruitment}

\section{Inclusion Criteria}

(1)Patients with breast cancer (Stage I-III), aged over 18 years;

(2)No recurrence and no tumor metastasis;

(3) Radiotherapy/chemotherapy was completed within 4-18 months after surgery.

\section{Exclusion Criteria}

(1) Language disorders or communication disorders;

(2) Serious exercise contraindications, such as cardiovascular or articular problems;

(3) Having any other serious illness or mental illness;

(4) Having received Qigong exercise or other planned exercise;

(5) Unable to use the Internet by oneself or with the help of others.

\section{Recruiting Methods}

This study intends to recruit 100 subjects from Shandong province who meet the above standard through doctor recommendation or through newspapers, TV and the Internet. 


\section{Subject Grouping}

Participants were briefed on the research and informed of the benefits and possible risks of clinical trials. They volunteered to participate in the study and completed an informed consent form. Subjects could withdraw from the study at any time. After the completion of informed consent, subjects were assigned to either the Qigong group or the regular exercise rehabilitation group by way of a random number table. Both groups received supervised exercise twice a week and were encouraged to volunteer for 3-4 unsupervised exercise sessions per week.

\section{Qigong Group}

Subjects in the Qigong group conducted the Baduanjin through live video streaming under the guidance of professors and professionals. Baduanjin is one of the most widely circulated Qigong methods, which is a traditional sport with a combination of physical activity, breathing, and psychological adjustment. The Baduanjin consists of eight movements, most of which are based on the horse stance combining movements of the upper limbs, head and neck and spine, as well as organized breathing. In the first week, Baduanjin was taught by qualified Qigong teaching staff through remote video, allowing the subjects to learn to master all movements of Baduanjin. Starting from week 2, subjects, led by a professional, performed Baduanjin twice a week, 30 minutes each, including five minutes of warming-up and coolingdown activities before and after the exercise. Participants were encouraged to actively practice Baduanjin during the rest of the time. Subjects were be required to report their practice experience, possible adverse reactions and side effects on a weekly basis, either orally or in writing. Subjects who exited the researcher were asked to explain the reason for the exit.

\section{Regular exercise rehabilitation group}

The control group adopted a combination exercise of walking and resistance training [7]. Subjects underwent a 30-minute resistance band training twice a week with a professional video tutorial, including 5 minutes of warm-up and cooling-down activities before and after the exercise. Subjects were encouraged to do walking exercise in the rest of the time, and the intensity of walking was determined based on pre-measured levels of aerobic capacity. The resistance load was determined based on the level of muscle strength measured in advance.

\section{Participant timeline}

Fig. 2 shows details on the schedule of enrollment, interventions, and assessments.

\section{Outcomes}

All data were measured and recorded 12 weeks after the baseline and intervention. The primary outcomes are quality of life which will be evaluated by using 36-Item Short-Form Health Survey(SF-36) and Functional Assessment of Cancer Therapy-Breast (FATC-B), and the second indicator is the level of physical fitness, including cardiopulmonary endurance, muscle strength, and flexibility. 


\section{Quality Of Life (QOL)}

\section{SF-36}

Sf-36 [16-17] is a general scale for evaluating health status, and its reliability and validity have been well recognized. The questionnaire included eight dimensions: physiological function (PF), role physical (RP), bodily pain (BP), general health (GH), vitality (VT), social function (SF), role emotional (RE) and mental health $(\mathrm{MH})$.

\section{FACT-B (Version 4.0)}

FACT-B (version 4.0)[18-19] is a targeted scale of quality of life for breast cancer survivors. It is recognized for good reliability, validity, and reactivity. FACT-B (version 4.0) includes five dimensions: physiological well-being (7 items), social/family well-being (7 items), emotional well-being (6 items), functional well-being (7 items), and additional concerns (9 items).

\section{Outcomes of physical fitness}

\section{Cardiopulmonary Endurance}

Bruce scheme was adopted and cycle ergometer was used for testing.

\section{Upper-limb strength test (Dumbbell Brachiocyrtosis Test)}

Subjects were asked to sit on a 44CM high-backed chair placed against the wall, hold a 5-pound dumbbell, extend their arms to the side of the chair naturally. Then they were asked to bend their arms upward to the maximum extent, then gradually swivel them outward to the palm up, and then put arms back to the side of the chair. The number of repetitions within 30 seconds was recorded.

\section{Lower-limb strength test (Sitting and Standing test)}

For safety, the straight-back chair (or folding chair) was placed against the wall. Subjects sat in the middle of a chair with their feet shoulder-width apart, slightly forward and backward and crossed arms close to the chest. During the test, the subjects stood up completely, then sat down completely. The number of times the subjects sat down in a chair within 30 seconds was recorded. For safety or requirement, the tester can use the arm for protective assistance [20].

\section{Body fat composition}

The percentage of body fat will be measured by skinfold thickness method. The measurement included triceps $\square$ anterior suprailiac and thigh.[21]

Flexibility (Hand Backward Stretch Test) 
The subject's hand was curled around the ipsilateral shoulder, stretching as far as possible into the middle of the back, with the palm close to the body. Subjects put the other hand behind the back, palm outward, stretched as far up as possible, and tried to touch or overlap the middle finger of both hands. The distance between the two middle fingers was measured twice in the vertical direction with an accuracy of $0.1 \mathrm{~cm}$. If the fingertips just touch, the score is zero. If they were not touched, then a negative distance is gotten, like -5 centimeters / -2 inches; If two fingers overlap, then a positive distance is acquired, such as $+2.5 \mathrm{~cm} /+1$ inch; Finally, the best experimental results could be acquired. When the subject is in pain during the test, the test shall stop immediately.

\section{Data collection and management}

In order to collect data in an accurate and scientific way, we trained personnel in charge of data measurement and collection with operational specifications. During the experimental data collection process, the subject's physical condition was closely observed and if the subject appeared any discomfort, the test would immediately stop, and the subjects would timely consult a doctor.

Monitoring was carried out by personnel independent of investigators and sponsors, including checking all informed consent and verifying the integrity of all data and source data. They would collect data and record on standard reports, and when access was complete, all recorded data were transmitted to a webbased data system through multiple inputs. All errors were crossed out with the researcher's signature and date. Any participant could withdraw from this study at any time for any reason.

\section{Statistical analysis}

The experimental results used mixed effect model [22] to compare the two sets of data. The random effect of the participants was evaluated, and the data were adjusted according to the random group, and the relevant baseline variables were used to analyze the data without adjusting the multiple comparisons. The effect differences between the comparison groups were calculated (expressed as effect values), and the results were expressed as inter-group differences (95\% confidence intervals). All analyses were performed using Stata 13.1 or the updated version.

\section{Blind Method}

After completing the baseline survey and physical fitness test, the computer random list generated by SPSS 16.0 software was used for randomization. Eligible patients were randomly divided into the experimental group and the control group, with a ratio of 1:1[23]. The group numbers were enclosed in a carbon-free envelope. The envelopes were kept by the research manager, who was not directly involved in the recruitment or follow-up of any participants, and the group number would then be published. Different people would register as participants and be assigned for intervention, and the results were kept by the research designer until the end of the experiment[24].

\section{Sample Size}


The sample size was estimated based on our previous test results [25]. The sample size of this experiment was estimated by the non-inferior test. The non-inferiority margin was 6 and the standard deviation was 10.31. According to the formula, each group should have 44 participants by formulating the inspection level (0.025) and the power of test 1- $\beta(0.2)$. During baseline inspection and follow-up, 50 participants in each group should participate in the experiment, given a $15 \%$ expulsion rate.

\section{Discussion}

The effect of Qigong intervention on postoperative rehabilitation of breast cancer has been confirmed by randomized controlled trials [26]. However, there are some problems in the traditional outpatient exercise rehabilitation, such as traffic, time consumption, space occupation, etc., which will affect the patients' enthusiasm to participate in rehabilitation to a certain extent, and also increases the medical economic burden. The development of Internet technology makes it possible to guide the rehabilitation of remote exercise. Based on this, we designed a randomized controlled trial with a sufficient sample size to verify the rehabilitation effect of remote guidance intervention of Qigong on postoperative breast cancer patients and to compare the effects of Qigong intervention and traditional exercise rehabilitation.

In addition, most of the study of exercise rehabilitation takes the improvement of patients' physical fitness level as the main research outcomes, which neglects the patient's subjective feeling and quality of life to some extent. Therefore, the quality of life of breast cancer patients after surgery is set as the first research indicator, followed by the related physical fitness outcomes.

Also, it is not clear whether conventional exercise intervention or Qigong intervention can prevent recurrence in breast cancer patients. To answer this question, a follow-up study of larger samples and longer periods of time are necessary. Due to the limitation of research funding, The exercise intervention and follow-up time are relatively short which makes it hard to study the recurrence of tumor in depth. If possible, subsequent studies will look at whether Qigong can prevent breast cancer recurrence.

\section{Declarations}

\section{Ethical Approval and Consent to participate}

Ethics approval and consent to participate

Ethical approval was granted by the Ethics Committee of The First Affiliated Hospital of Shandong First Medical University and was retrospectively registered in Chinese Clinical Trial Registry (registration number:ChiCTR1900027989). Only the participants who signed the in- formed consent document were included in the study.

\section{Acknowledgements}


We would like to acknowledge Open project of Sino US sports economic and health engineering cooperation research center in Shandong Provincefor the contribution in funding the study. In addition, we want to express our appreciation to all participants who made their best efforts to participate actively in this study.

\section{Trial status}

At the time of submission, our recruitment was in progress. The test was carried out on May 1, 2020.Approved by the ethics committee, No. [2019] LSZ (S175), medical ethics committee of the First Affiliated Hospital of Shandong First Medical University, October 9, 2019. The main recruitment phase of the trial started on May 1, 2020 (The trial is at Version 1, January 2020). The test is scheduled to end on May 1, 2021. Plan for 12 months.

\section{Authors' contributions}

MD initiated the idea and design of the study. XD and LY authored the initial draft, deployed personnel in the process of experiment and revised the final draft. KY recruited participants. MD was involved in initial conception of the project design. YS performed the study intervention. XY and YL performed the collection of data. All authors gave final approval and agreed to be accountable for all aspects of the work.

\section{Funding}

This study received funding from Open project of Sino US sports economic and health engineering cooperation research center in Shandong Provincefor.

Funding agencies are not involved in the design of the study and collection, analysis, and interpretation of data and in writing the manuscript.

\section{Consent for publication}

Not applicable.

\section{Competing interests}

Lina Yu,Xiaosheng Dong, Kai Yuan,Xiangren Yi, Yuanlong Shen,Meng Ding have stated explicitly that there is no conflict of interest in connection with this article.

\section{Author details}

1 College of Physical Education, Shandong Normal University, Jinan, 250014, China;2 College of Physical Education, Shandong University, Jinan, 250011, China;3 The first affiliated hospital of shandong first medical university,Jinan, 250011, China.

\section{Abbreviations}


Sf-36 the Mos 36-item Short Form Health Survey;PF:physiological function ;

RP:role physical ;BP: bodily pain ;GH:general health; VT:vitality; SF:social function;RE:role emotional;MH:mental health.

\section{References}

1.Watters, D. A. (2017). The World Health Organization Surgical Safety Checklist. Anz Journal of Surgery, 87(12), 961-962. doi:10.1111/ans.14210

2.Berry, D. A., Cronin, K. A., Plevritis, S. K., Fryback, D. G., Clarke, L., Zelen, M., . . Surveillance Modeling Network, C. (2005). Effect of screening and adjuvant therapy on mortality from breast cancer. N Engl J Med, 353(17), 1784-1792. doi:10.1056/NEJMoa050518

3.Welch, H. G., Prorok, P. C., O'Malley, A. J., \& Kramer, B. S. (2016). Breast-Cancer Tumor Size, Overdiagnosis, and Mammography Screening Effectiveness. New England Journal of Medicine, 375(15), 1438-1447. doi:10.1056/NEJMoa1600249

4.McNeely, M. L., Campbell, K. L., Rowe, B. H., Klassen, T. P., Mackey, J. R., \& Courneya, K. S. (2006). Effects of exercise on breast cancer patients and survivors: a systematic review and meta-analysis. Canadian Medical Association Journal, 175(1), 34-41. doi:DOI 10.1503/cmaj.051073

5.Kim, S., Ko, Y. H., Song, Y., Kang, M. J., Lee, H., Kim, S. H., . . Han, J. (2019). Development of an exercise adherence program for breast cancer survivors with cancer-related fatigue-an intervention mapping approach. Supportive Care in Cancer, 27(12), 4745-4752. doi:10.1007/s00520-019-04785-2

6.Yang, A., Sokolof, J., \& Gulati, A. (2018). The effect of preoperative exercise on upper extremity recovery following breast cancer surgery: a systematic review. International Journal of Rehabilitation Research, 41(3), 189-196. doi:10.1097/Mrr.0000000000000288

7.Lee, T. I., Chen, H. H., \& Yeh, M. L. (2006). Effects of Chan-Chuang Qigong on improving symptom and psychological distress in chemotherapy patients. American Journal of Chinese Medicine, 34(1), 37-46. doi:Doi 10.1142/S0192415x06003618

8.Dong, X. S., Yi, X. R., Huang, S. Y., Gao, D. Z., Chao, M. Y., \& Ding, M. (2018). The effects of combined exercise intervention based on Internet and social media software for postoperative patients with breast cancer: study protocol for a randomized controlled trial. Trials, 19. doi:ARTN 47710.1186/s13063-0182857-3

9.Huang, S. M., Tseng, L. M., Chien, L. Y., Tai, C. J., Chen, P. H., Hung, C. T., \& Hsiung, Y. (2016). Effects of non-sporting and sporting qigong on frailty and quality of life among breast cancer patients receiving chemotherapy. European Journal of Oncology Nursing, 21, 257-265. doi:10.1016/j.ejon.2015.10.012 
10.Oh, B., Butow, P. N., Mullan, B. A., Clarke, S. J., Beale, P. J., Pavlakis, N., . . Vardy, J. (2012). Effect of medical Qigong on cognitive function, quality of life, and a biomarker of inflammation in cancer patients: a randomized controlled trial. Supportive Care in Cancer, 20(6), 1235-1242. doi:10.1007/s00520-0111209-6

11.Lee, T. I., Chen, H. H., \& Yeh, M. L. (2006). Effects of Chan-Chuang Qigong on improving symptom and psychological distress in chemotherapy patients. American Journal of Chinese Medicine, 34(1), 37-46. doi:Doi 10.1142/S0192415x06003618

12.Chen, P. H., Huang, S. M., Tai, C. J., Chien, L. Y., Lien, P. J., \& Chen, Y. H. (2015). Meditative qigong relieved symptom severity and interference among patients with breast carcinoma receiving chemotherapy. European Journal of Integrative Medicine, 7(6), 617-622. doi:10.1016/j.eujim.2015.08.010

13.Larkey, L., Huberty, J., Pedersen, M., \& Weihs, K. (2016). Qigong/Tai Chi Easy for fatigue in breast cancer survivors: Rationale and design of a randomized clinical trial. Contemporary Clinical Trials, 50, 222-228. doi:10.1016/j.cct.2016.08.002

14.Liu, W., Schaffer, L., Herrs, N., Chollet, C., \& Taylor, S. (2015). Improved sleep after Qigong exercise in breast cancer survivors: A pilot study. Asia Pac J Oncol Nurs, 2(4), 232-239. doi:10.4103/23475625.170537

15.Chan, C. L., Wang, C. W., Ho, R. T., Ho, A. H., Ziea, E. T., Taam Wong, V. C., \& Ng, S. M. (2012). A systematic review of the effectiveness of qigong exercise in cardiac rehabilitation. Am J Chin Med, 40(2), 255-267. doi:10.1142/S0192415X12500206

16.Larkey, L. K., Roe, D. J., Smith, L., \& Milistine, D. (2016). Exploratory outcome assessment of Qigong/Tai Chi Easy on breast cancer survivors. Complementary Therapies in Medicine, 29, 196-203. doi:10.1016/j.ctim.2016.10.006

17.Chang, J. H., \& Guo, J. T. (2015). Treatment of chronic hepatitis B with pattern recognition receptor agonists: Current status and potential for a cure. Antiviral Research, 121, 152-159. doi:10.1016/j.antiviral.2015.07.006

18.Imayama, I., Alfano, C. M., Neuhouser, M. L., George, S. M., Smith, A. W., Baumgartner, R. N., . . . McTiernan, A. (2013). Weight, inflammation, cancer-related symptoms and health-related quality of life among breast cancer survivors. Breast Cancer Research and Treatment, 140(1), 159-176. doi:10.1007/s10549-013-2594-y

19.Niu, L. S., Liang, Y. C., \& Niu, M. E. (2019). Factors influencing fear of cancer recurrence in patients with breast cancer: Evidence from a survey in Yancheng, China. Journal of Obstetrics and Gynaecology Research, 45(7), 1319-1327. doi:10.1111/jog.13978 
20.Nakamura, K., Nagasawa, Y., Sawaki, S., Yokokawa, Y., \& Ohira, M. (2016). Effect of Different Seat Heights during an Incremental Sit-To-Stand Exercise Test on Peak Oxygen Uptake in Young, Healthy Women. Journal of Sports Science and Medicine, 15(3), 410-416.

21.ACSM's Guide for Exercise Testing and Prescription by American College of Sports medicine-Ninth Edition

22.ong, X. S., Yi, X. R., Huang, S. Y., Gao, D. Z., Chao, M. Y., \& Ding, M. (2018). The effects of combined exercise intervention based on Internet and social media software for postoperative patients with breast cancer: study protocol for a randomized controlled trial. Trials, 19. doi:ARTN 477

$10.1186 / \mathrm{s} 13063-018-2857-3$

23.Roy, M., Williams, S. M., Brown, R. C., Meredith-Jones, K. A., Osborne, H., Jospe, M., \& Taylor, R. W. (2018). High-Intensity Interval Training in the Real World: Outcomes from a 12-Month Intervention in Overweight Adults. Medicine and Science in Sports and Exercise, 50(9), 1818-1826.

doi:10.1249/Mss.0000000000001642

24.Klein, D., Mercier, M., Abeilard, E., Puyraveau, M., Danzon, A., Dalstein, V., ... Velten, M. (2011). Longterm quality of life after breast cancer: a French registry-based controlled study. Breast Cancer Research and Treatment, 129(1), 125-134. doi:10.1007/s10549-011-1408-3

25.Xiaosheng, D., Xiangren, Y., Shuyuan, H., Dezong, G., Mengyao, C., \& Meng, D. (2018). The effects of combined exercise intervention based on Internet and social media software for postoperative patients with breast cancer: study protocol for a randomized controlled trial. Trials, 19(1), 477.

doi:10.1186/s13063-018-2857-3

26.Larkey, L. K., Roe, D. J., Smith, L., \& Milistine, D. (2016). Exploratory outcome assessment of Qigong/Tai Chi Easy on breast cancer survivors. Complementary Therapies in Medicine, 29, 196-203. doi:10.1016/j.ctim.2016.10.006

\section{Figures}




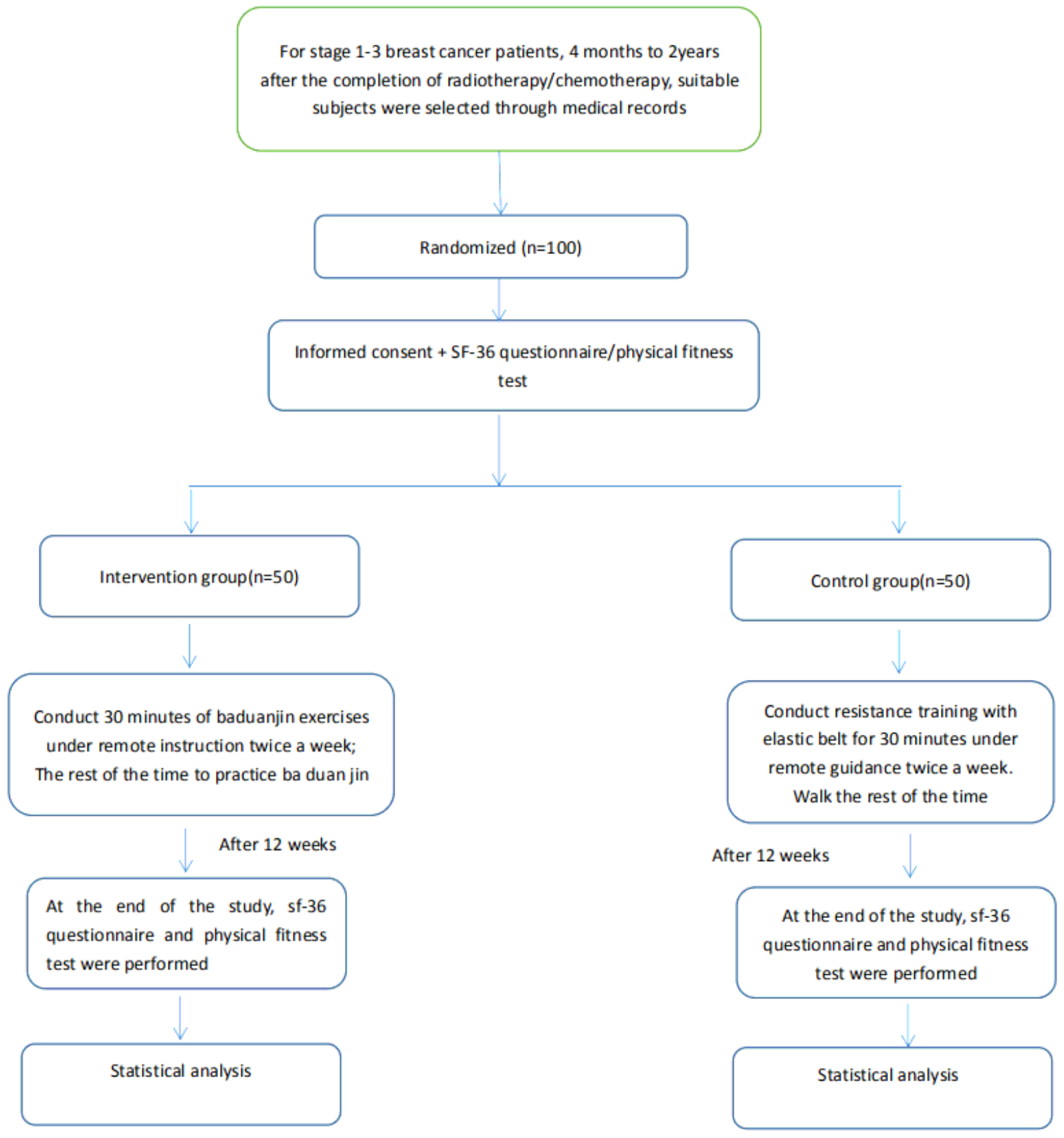

The flow chart of the trial design

\section{Figure 1}

The flow chart of the trial design 


\begin{tabular}{|c|c|c|c|c|c|c|c|c|c|c|c|c|c|}
\hline \multirow[b]{3}{*}{ TIMEPOINT } & \multicolumn{12}{|c|}{ Study period } & \multirow{3}{*}{\begin{tabular}{|l}
$\begin{array}{l}\text { Close } \\
\text {-out }\end{array}$ \\
$12-$ \\
keek \\
\end{tabular}} \\
\hline & \multicolumn{2}{|c|}{ Baseline } & \multicolumn{10}{|c|}{ Post-allocation } & \\
\hline & $\begin{array}{l}0- \\
\text { weel }\end{array}$ & $\begin{array}{l}1- \\
\text { week }\end{array}$ & $\begin{array}{l}2- \\
\text { week }\end{array}$ & $\begin{array}{l}3- \\
\text { week }\end{array}$ & $\begin{array}{l}4- \\
\mathrm{k} \text { week }\end{array}$ & $\begin{array}{l}\text { 5- } \\
\text { k week }\end{array}$ & $\begin{array}{l}6- \\
\text { week }\end{array}$ & $\begin{array}{l}7- \\
\text { week }\end{array}$ & $\begin{array}{l}8- \\
\text { week }\end{array}$ & $\mathrm{k}$ week & $\begin{array}{l}10^{-} \\
\text {week }\end{array}$ & $\mid \begin{array}{l}11- \\
\text { week }\end{array}$ & \\
\hline \multicolumn{14}{|l|}{ ENROLLMENT: } \\
\hline \multicolumn{14}{|c|}{ Elgibilit screen $\mathbf{X}$} \\
\hline \multicolumn{14}{|c|}{ Informed consent $\times$} \\
\hline Examination & & $x$ & & & & & & & & & & & \\
\hline Randomization & & $x$ & & & & & & & & & & & \\
\hline \multicolumn{14}{|l|}{ INTERVENTIONS: } \\
\hline $\begin{array}{l}\text { Diversity } \\
\text { exercise } \\
\text { intervention }\end{array}$ & & $x$ & $x$ & $x$ & $x$ & $x$ & $x$ & $x$ & $x$ & $x$ & $x$ & $x$ & \\
\hline $\begin{array}{l}\text { Normal treatment } \\
\text { and } \\
\text { rehabilitation } \\
\text { according to } \\
\text { daily } \\
\text { specifications } \\
\text { of the hospital }\end{array}$ & & $x$ & $x$ & $x$ & $x$ & $x$ & $x$ & $x$ & $x$ & $x$ & $x$ & $x$ & \\
\hline \multicolumn{14}{|l|}{ ASSESSMENTS: } \\
\hline SF- -36 & & $x$ & & & & & & & & & & & $x$ \\
\hline PROMIS & & $x$ & & & & & & & & & & & $x$ \\
\hline Physical fitness & & $x$ & & & & & & & & & & & $x$ \\
\hline \multicolumn{14}{|l|}{$\begin{array}{l}\text { PARTICIPANT } \\
\text { SAFETY: }\end{array}$} \\
\hline Adverse events & & $x$ & $x$ & $x$ & $x$ & $x$ & $x$ & $x$ & $x$ & $x$ & $x$ & $x$ & \\
\hline
\end{tabular}

Figure 2

Recommendations for Interventional Trials (SPIRIT) diagram of enrolment, treatment, and assessments over time

\section{Supplementary Files}


This is a list of supplementary files associated with this preprint. Click to download.

- spirit.doc 